\title{
Breeding potential of maize germplasm line GEMS-0067 for high amylose proportion
}

\author{
Nan Han ${ }^{1}$, Wanchen Li ${ }^{1}$, Chuanxiao Xie ${ }^{2,3,}$, Fengling Fu ${ }^{1, *}$
}

1 Maize Research Institute, Sichuan Agricultural University, Chengdu 6111130, China; 635460761@qq.com (N.H.); aumdyms@sicau.edu.cn (W.L.); ffl@sicau.edu.cn (F.F.)

${ }^{2}$ Institute of Crop Sciences, Chinese Academy of Agricultural Sciences, Beijing 100081, China; xiechuanxiao@caas.cn (C.X.)

${ }^{3}$ National Key Facility for Crop Gene Resources and Genetic Improvement, Beijing, 100081, China; xiechuanxiao@caas.cn (C.X.)

* Correspondence: xiechuanxiao@caas.cn (C.X.), Tel.: +86-10-82106748; ffl@sicau.edu.cn (F.F.); Tel.: $+86-28-86290912$

\begin{abstract}
Maize varieties with high amylose proportion are more valuable for starch industry. The SBE IIb gene encodes one of the starch branching isozymes (SBEI, SBEIIa, and SBEIIb). Its recessive mutant amylose-extender ( $a e / s b e 2 b$ ) decreases the total activities of SBEs and increases amylose proportion up to $60 \%$. Here, the breeding potential of introduced germplasm line GEMS-0067 was evaluated by genotyping and phenotyping. The deletion of the ninth exon of the SBE IIb gene, high amylose proportion, and the typical irregular granules suggested that this germplasm line was derived from the same resource of high amylose line AE11. The gelatinization and thermal properties, and degree of polymerization of starch chain showed its advantages used for high amylose breeding. However, the negative correlation between amylose proportion and starch content, as well as ker-nel filling characteristics should be overcome during breeding process.
\end{abstract}

Keywords: ae mutant; amylose; breeding potential; maize; SBEIIb

\section{Introduction}

Maize is one of the staple crops in the world [1]. The kernels provide large amount of starch, consisting of essentially linear amylose and highly branched amylopectin [2]. The content and proportion of amylose and amylopectin determine the quality and usage of maize [3]. Native starch granules in cereal endosperm normally comprise $20 \%-30 \%$ amylose and 70\%-80\% amylopectin [4]. In waxy maize, which are usually planted as vegetable varieties for favorite food, the proportion of amylopectin can be as high as $100 \%$ almost $[5,6]$. Whereas, high amylose varieties with amylose proportion more than $50 \%$ are more valuable for starch industry and beneficial to control dietary intake [7-9], because it has high level of resistance to gelatinization and hydrolysis and cannot be digested in the upper gastrointestinal tract $[8,10-13]$. Therefore, maize improvement for high amylose proportion has attracted more considerable attention in recent years [14-16].

The amylose proportion in maize endosperm is controlled by a series of starch synthesis genes $[4-6,12,17]$. One of the critical is the $S B E I I b$ gene that encodes starch branching enzyme (SBE) IIb (one of the SBE isozymes: SBEI, SBEIIa, and SBEIIb). The full length of its genomic sequence is 23,449 bp and contains 22 exons [4-6, 12, 16-19]. The amylose proportion of its recessive mutant amylose-extender (ae/sbe $2 b$ ) is over 60\% [20-24]. For example, the total activities of SBEs in an ae mutant AE11 were decreased to about $71 \%$ of its wild control because of the deletion of the ninth exon of the SBEIIb gene containing 84 
bases. Under the modification of other modifier genes, such as sbe1, the amylose proportion of this line was raised as high as 70\% [5].

In the present study, the introduced germplasm line GEMS-0067 released as parent of maize breeding for high amylose by Truman State University [25], together with its wild type (WT) control, was genotyped for the $a e / s b e 2 b$ mutation, and phenotyped for morphology of starch granules, starch content and amylose proportion, gelatinization and thermal properties, degree of polymerization, and major agronomic traits, to evaluate its breeding potential for high amylose proportion.

\section{Results}

\subsection{The ae/sbe $2 b$ gene}

A 750 and a 1576 bp specific band was separated from the amplified products of GEMS-0067 and its WT control (Figure 1A). The results of sequencing and alignment showed that the full length of the genomic sequence of the ae/SBE IIb gene was $23449 \mathrm{bp}$ in the WT control. The whole 84 bases of the ninth exon were completely deleted in GEMS0067, but not causing a frame shift (Figure 1B).

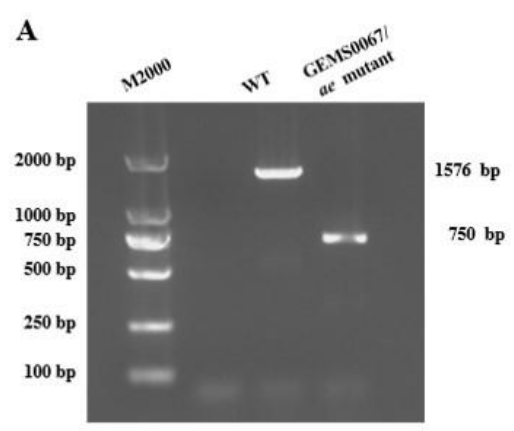

B

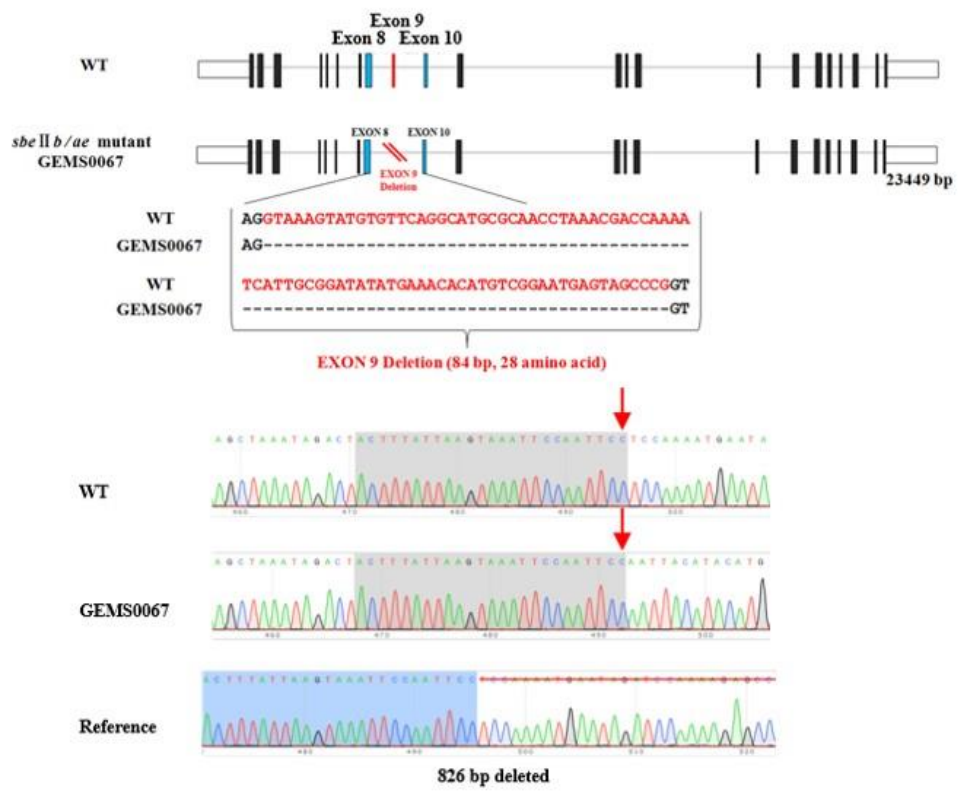

Figure 1. The $a e / s b e 2 b$ gene in inbred germplasm line GEMS-0067. (A) Specific bands separated from the amplified products of GEMS0067 and the WT. (B) The structure pattern of the genomic sequences of the ae/SBEIIb gene between GEMS-0067 and WT.

\subsection{Morphology of starch granules and amylose proportion}

The scanning electron microscopy images showed that the starch granules isolated from WT endosperm were spherical and elliptical with smooth surface, whereas the starch of GEMS0067 contained individual or aggregate elongated granules with irregular depressions, as well as small subgranules (Figure 2). The starch content and amylose proportion of GEMS0067 were $72.63 \%$ and $72.95 \%$, respectively, significantly different from $74.21 \%$ and $26.26 \%$ of WT (Table 1 ).

Table 1. Starch content and amylose proportion of GEMS0067 and WT.

\begin{tabular}{ccc}
\hline & GEMS0067 & WT \\
\hline Starch content & $72.63 \% *$ & $74.21 \%$ \\
Amylose proportion & $72.95 \% *$ & $26.26 \%$ \\
\hline
\end{tabular}

\footnotetext{
${ }^{1}$ Values are presented as mean \pm standard deviation $(\mathrm{n}=3)$. The asterisk $\left(^{*}\right)$ and double asterisk $\left(^{* *}\right)$ indicate significance at $\mathrm{p} \leq 0.05$ and $\mathrm{p} \leq 0.01$ respectively.
} 

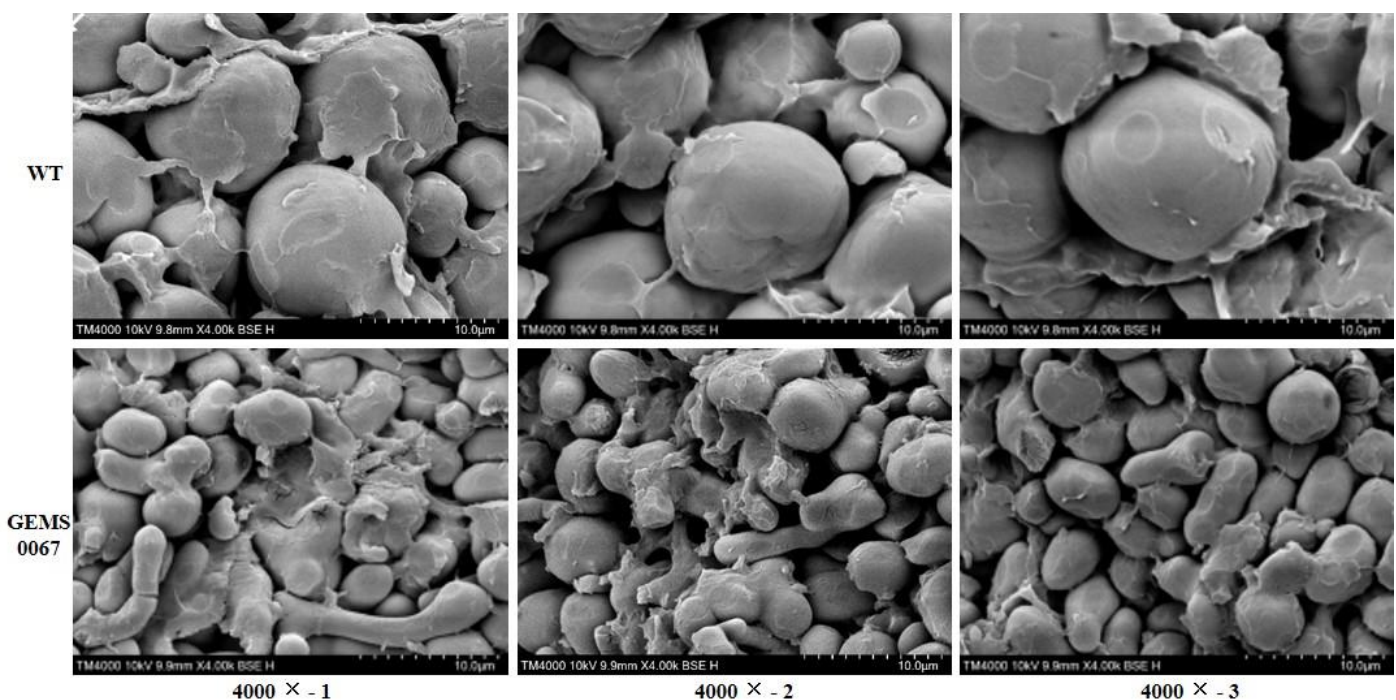

Figure 2. SEM images of starch granules of GEMS0067 and WT. The starch granules of WT were spherical and elliptical with smooth surface. The starch granules of GEMS0067 were elongated with irregular depressions, as well as more small subgranules..

\subsection{Gelatinization properties}

Rapid viscosity analysis showed obvious difference of viscosity curves between GEMS0067 and WT starch (Figure 3). Along with the increasing of temperature, the viscosity of WT starch gradually increased caused by gelatinization and presented a typical double-peak curve, whereas GEMS0067 demonstrated almost a horizontal straight line with a small peak at the beginning. The peak viscosity, through viscosity, break viscosity, final viscosity, and setback viscosity of GEMS0067 starch were significantly lower, while its peak time and gelatinization temperature were higher than those of WT starch (Table 2).

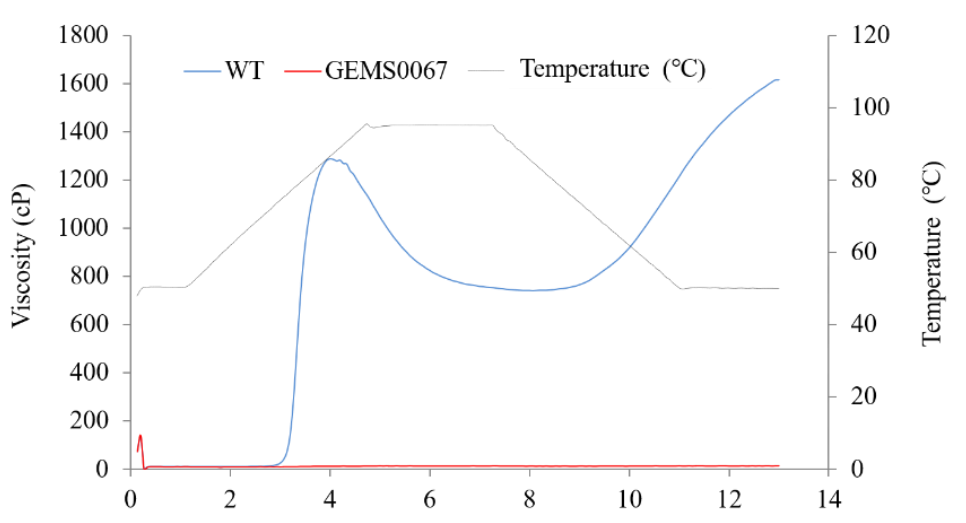

Figure 3. Viscosity curves of GEMS0067 and WT starch. The WT starch presented a typical double-peak curve, whereas GEMS0067 starch demonstrated almost a horizontal straight line with a small peak at the beginning.

Table 2. Gelatinization properties of starch of GEMS0067 and WT

\begin{tabular}{ccc}
\hline Property & GEMS0067 & WT \\
\hline Peak viscosity $(\mathrm{cP})$ & $13.67 \pm 1.15^{* *}$ & $1256.67 \pm 32.02$ \\
Through viscosity $(\mathrm{cP})$ & $11.67 \pm 1.15^{* *}$ & $722.33 \pm 23.46$ \\
Breakdown viscosity $(\mathrm{cP})$ & $2.00 \pm 0.00^{* *}$ & $534.33 \pm 10.97$ \\
Final viscosity $(\mathrm{cP})$ & $13.00 \pm 1.73^{* *}$ & $1575.33 \pm 37.69$ \\
Setback viscosity $(\mathrm{cP})$ & $1.33 \pm 0.58^{* *}$ & $853.00 \pm 37.24$ \\
Peak time $(\mathrm{min})$ & $4.56 \pm 0.41^{* *}$ & $4.02 \pm 0.04$ \\
Gelatinization temperature $\left({ }^{\circ} \mathrm{C}\right)$ & $>95$ & $75.18 \pm 0.03$ \\
\hline
\end{tabular}

\footnotetext{
${ }^{1}$ Values are presented as mean \pm standard deviation $(n=3)$. cP centipoise. Double asterisk $\left.{ }^{* *}\right)$ indicates significant difference at $\mathrm{P}<$
} 0.01 . 


\subsection{Thermal properties}

Differential scanning calorimetry showed that the transition temperatures, including onset temperature (To), peak temperature (Tp) and conclusion temperature (Tc), of GEMS0067 starch were significantly higher than those of WT starch, and the gelatinization enthalpy of GEMS0067 starch was significantly lower than that of WT starch (Table 3). Along with the increasing of temperature, the differential scanning calorimetry thermogram of WT starch presented an obvious absorption peak at $70^{\circ} \mathrm{C}$, whereas the absorption peak of GEMS0067 starch almost became a straight line (Figure 4) because its gelatinization enthalpy was six times lower than WT (Table 3).

Table 3. Thermal properties of starch of GEMS0067 and WT

\begin{tabular}{crc}
\hline Property & GEMS0067 & \multicolumn{1}{c}{ WT } \\
\hline Onset temperature $\left({ }^{\circ} \mathrm{C}\right)$ & $73.38 \pm 0.93^{* *}$ & $65.19 \pm 0.15$ \\
Peak temperature $\left({ }^{\circ} \mathrm{C}\right)$ & $91.65 \pm 2.97^{* *}$ & $70.09 \pm 0.03$ \\
Conclusion temperature $\left({ }^{\circ} \mathrm{C}\right)$ & $101.05 \pm 0.09^{* *}$ & $76.21 \pm 0.13$ \\
Gelatinization enthalpy $\left(\mathrm{J} \cdot \mathrm{g}^{-1}\right)$ & $2.33 \pm 0.54^{* *}$ & $12.60 \pm 0.31$ \\
\hline
\end{tabular}

${ }^{1}$ Values are presented as mean \pm standard deviation $(n=3)$. Double asterisk $\left.{ }^{* *}\right)$ indicates significant difference at $\mathrm{P}<0.01$.

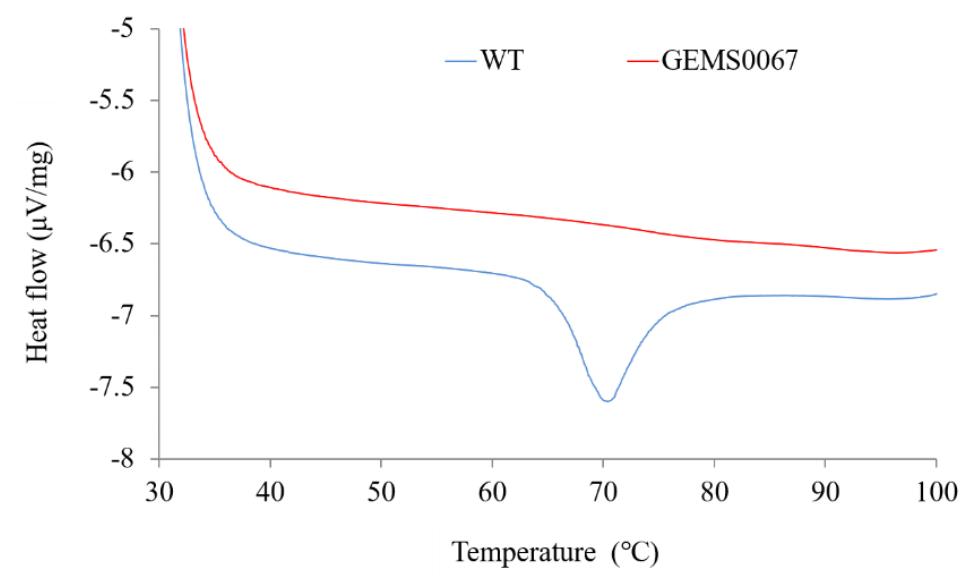

Figure 4. Differential scanning calorimetry thermogram of GEMS0067 and WT starch. The thermogram of WT starch presented an obvious absorption peak at $70^{\circ} \mathrm{C}$, whereas GEMS0067 starch almost became a straight line. 


\subsection{Degree of polymerization}

The result of high-performance anion-exchange chromatography (HPAEC) showed that the degree of polymerization heavily depended on the proportion of amylose and amylopectin of starch. Referring to Hanashiro et al. (1996) [26], the percentage of short chains $(6 \leq \mathrm{DP} \leq 24)$ was less in GEMS0067 starch than that in WT starch, whereas the percentage of medium chains $(25 \leq \mathrm{DP} \leq 36)$ and long chains $(\mathrm{DP} \geq 37)$ was more in GEMS0067 starch than that in WT starch (Figure 5).

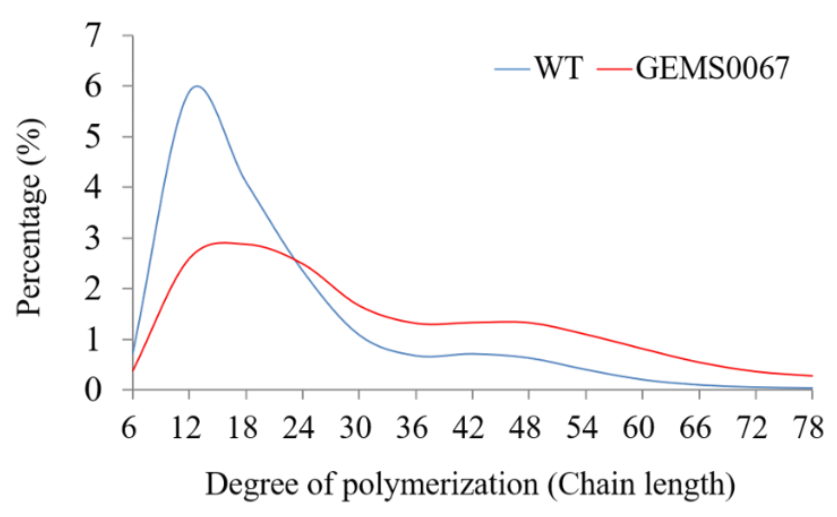

Figure 5. Degree of polymerization of GEMS0067 and WT starch. The percentage of short chains $(6 \leq \mathrm{DP} \leq 24)$ was less, and the percentage of medium chains $(25 \leq \mathrm{DP} \leq 36)$ and long chains $(\mathrm{DP} \geq 37)$ was more in GEMS0067 starch than that in WT starch.

\subsection{Agronomic traits}

During all the growth period, the two lines grew normally and was not infected by any pathogen. The result of paired $t$-test showed that the differences of anthesis stage, silking stage, growth period, plant height, ear height, and row number per ear were nonsignificant between GEMS-0067 and WT (Table 4). Whereas, the GEMS0067 kernels were a little shrunk and dull, and its endosperm was stained much darker by $\mathrm{I}_{2} / \mathrm{KI}$ solution than WT kernels (Figure 6), resulting in the significant decrease of its 100-kernel weight and kernel weight per plant, although its kernel number per row was significantly more than WT (Table 4).

Table 4. Agronomic traits and statistical significance

\begin{tabular}{crr}
\hline Trait & GEMS0067 & \multicolumn{1}{c}{ WT } \\
\hline Anthesis stage (d) & $56 \pm 0.6$ & $55 \pm 0.5$ \\
Silking stage (d) & $60 \pm 0.3$ & $59 \pm 0.4$ \\
Growth period (d) & $101 \pm 1.2$ & $100 \pm 1.2$ \\
Plant height (cm) & $154.3 \pm 5.4$ & $152.6 \pm 8.7$ \\
Ear height (cm) & $70.1 \pm 8.6$ & $70.7 \pm 5.6$ \\
Row number per ear & $15.0 \pm 0.6$ & $14.9 \pm 0.9$ \\
Kernel number per row & $28.0 \pm 1.2^{*}$ & $26.0 \pm 1.5$ \\
100-kernel weight $(\mathrm{g})$ & $25.6 \pm 0.1^{*}$ & $30.3 \pm 0.2$ \\
Kernel weight per ear $(\mathrm{g})$ & $107.5 \pm 0.6^{* *}$ & $117.3 \pm 1.0$ \\
\hline
\end{tabular}

${ }^{1}$ Values are presented as mean \pm standard deviation $(n=3)$. The asterisk $\left(^{*}\right)$ and double asterisk $\left({ }^{* *}\right)$ indicate significance at $\mathrm{p} \leq 0.05$ and $p \leq 0.01$ respectively. 


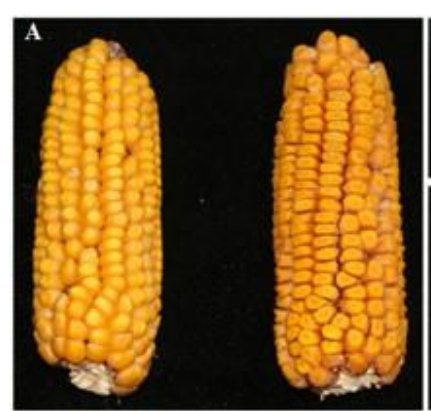

WT

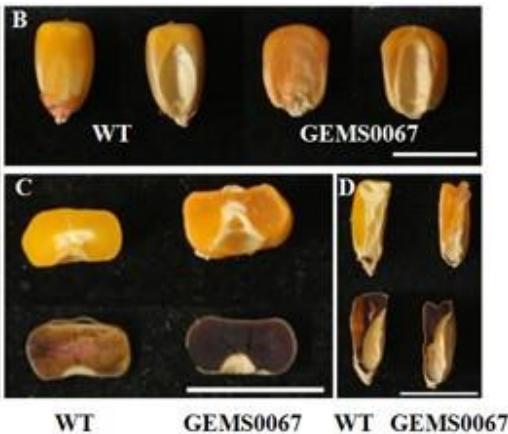

Figure 6. Shrunk kernels and darker stained endosperm of inbred germplasm line GEMS-0067. (A) and (B) The kernels of GEMS0067 were a little shrunk and dull. (C) and (B) The endosperm of GEMS0067 was stained much darker than WT. The wight bar is $1 \mathrm{~cm}$ long.

\section{Discussion}

The SBE IIb gene encodes one of the starch branching enzyme (SBE) isozymes (SBEI, SBEIIa, and SBEIIb) that play critical role in synthesis of amylopectin [4-6, 12, 16-22]. Its recessive mutant amylose-extender (ae/sbe2b) decreased the total activities of SBEs to about $71 \%$ and increases amylose proportion of maize kernel up to $60 \%$ [23-25]. From the $S B E I I b$ gene of the inbred germplasm line GEMS-0067, the whole 84 bases of the ninth exon were completely deleted (Figure 1B), suggesting that this germplasm line was derived from the same resource of high amylose inbred line AE11 [5].

The individual or aggregate elongated granules with irregular depressions and small subgranules (Figure 2), and high amylose proportion (Table 1), as well as the darker staining of the endosperm by $\mathrm{I}_{2} / \mathrm{KI}$ solution (Figure $6 \mathrm{C}$ ), are the typical phenotypes of the recessive mutant amylose-extender (ae/sbe2b) $[7,15,22,27,28]$. Those are caused by inhibition of SBE activity [28, 29].

The gelatinization temperature of GEMS0067 starch was recorded as $>95^{\circ} \mathrm{C}$ because it was higher than the upper limit of heating temperature of the differential scanning calorimeter. This property of maize amylose was also observed under confocal laser scanning microscopy and scanning electron microscopy [30]. The viscosity of starch was increased along with its gelatinization during gradual heating due to the absorption of water and loss of starch structure [31-33]. The significantly lower peak viscosity, through viscosity, break viscosity, final viscosity and setback viscosity, and gelatinization enthalpy (Figure 3, Table 2, 3), and significantly higher peak time, gelatinization temperature, and transition temperatures (To, Tp, and Tc, Table 2, 3), as well as the inconspicuous absorption peak (Figure 4) of GEMS0067 starch implied that gelatinization initiation of GEMS0067 starch needed much more energy to heat, because of its high proportion of amylose (Table 1) and high percentage of medium and long chains (Figure 5).

Negative correlation between amylose proportion and starch content, as well as kernel filling characteristics, was found in most of high amylose inbred germplasm lines [34]. In some high amylose lines with recessive mutation of some other modifier genes, the kernel filling characteristics were not much affected, but the amylose proportion was not as high as the $a e / s b e 2 b$ mutant lines [35]. In the present study, the shrinkage of GEMS0067 kernels resulted in the significant decrease of its 100-kernel weight (Figure 6, Table 4). However, the significant increase of kernel number per row recovered some of the loss. The kernel weight per ear of GEMS0067 was only 8.3\% lower than its WT (Table 4). Therefore, GEMS0067 was suggested as an elite germplasm line for maize breeding of high amylose proportion.

\section{Materials and Methods}

\subsection{Genotyping of ae/sbe2b mutation}

The genomic DNA was extracted from the leaf samples of the inbred germplasm line GEMS-0067 and its WT control with the CTAB buffer [36]. The quality and quantity of 
DNA samples were detected on Nano Photometer ${ }^{\mathrm{TM}}$ P-Class (Implen, Schatzbogen, Germany) and visualized by $1 \%$ agarose gel electrophoresis. The reference sequence of the WT SBE II b gene (GenBank: AF072725.1) was downloaded from the NCBI database (https://www.ncbi.nlm.nih.gov/gene/?term=AF072725.1). Referring to Kim et al. (1998) [20], the genomic sequence from the 8th to 10th exon of the SBE II b gene was amplified with a pair of specific primers (5'-CCAGCCTGGATCAAGTACTC-3'/5'-CTTGGATACAATGCAGTGCAA- $3^{\prime}$ ), separated by $1.5 \%$ agarose gel electrophoresis, subcloned into pMD-19-T vector, and sequenced at Sengon Biotech (China). The possible mutation of the ae/SBE II $b$ gene was genotyped by alignment between the inbred germplasm line GEMS0067 and its WT control by using the SnapGene software (https://www.snapgene.com/).

\subsection{Scanning electron microscopy}

As described by He et al. (2020) and Lin et al. (2016) [5, 8], starch was isolated from mature endosperm, dried completely at $40^{\circ} \mathrm{C}$, mounted on aluminum specimen holder using double-sided adhesive tape and sputtered with a gold in a vacuum evaporator, and viewed with a scanning electron microscopy (Hitachi, Tokyo, Japan). The images of starch granules were obtained at an accelerating voltage of $10 \mathrm{kV}$ and a magnification of $4000 \times$.

\subsection{Determination of starch content and amylose proportion}

According to the National Stardard for determination of crude starch in cereal seeds (National Bureau of Standards of the People's Republic of China 1985) [37], three replicates of kernel samples were wetted with calcium chloride acetic acid solution, hydrolyzed in a glycerin bath at $119 \pm 1{ }^{\circ} \mathrm{C}$ for $30 \mathrm{~min}$. After precipitating protein by addition of zinc sulfate and potassium ferrocyanide solution, the samples were made up to $100 \mathrm{ml}$ volume with distilled water, filtrated, and read for rotation angle in a polarimeter (MCP150, Anton Paar, China). Starch content was as:

$$
\text { Starch content }(\%)=\frac{\alpha \times 106}{\mathrm{~L} \times \mathrm{W}(100-\mathrm{H}) \times 203}
$$

Where, $\alpha, \mathrm{L}, \mathrm{W}, \mathrm{H}$, and 203 stand for the rotation angle, length $(\mathrm{dm})$ of the polarizing tube, weight of sample, moisture content of sample, and specific rotation of starch, respectively.

Amylose proportion of three replicates of each line was determined by using an amylose/amylopectin assay kit (Megazyme, Bray, Ireland) according to the manufacturer's instructions. After eliminating the possible lipids, proteins and amylopectin by using ethanol and concanavalin A, respectively [38], the remained amylose was enzymatically hydrolyzed to D-glucose, oxidized with glucose oxidase/peroxidase reagent, treated with the GOPOD reagent, and read for absorbance at $510 \mathrm{~nm}$ in a spectrophotometer (UV5Nano, Mettler Toledo, Switzerland).

\subsection{Determination of physical properties}

As described by Wang et al. (2018) [39], the starch samples were homogenized and sieved through a 200-mesh sifter, determined for moisture content in a rapid moisture meter (HR83-P, Mettler Toledo, Switzerland), accurately weighted for $5 \mathrm{~g}$ (error $<0.05 \mathrm{~g}$ ), transferred onto the aluminum foil, mixed well with $25 \mathrm{ml}$ sterile water, and determined the viscosity in a rapid viscosity analyzer (RVA Super 4, Newport Scientific, Australia).

As described by Wang et al. (2018) [39], the homogenized and sieved starch samples were accurately weighted for $10 \mathrm{mg}$, add with $30 \mu \mathrm{L}$ sterile water, balanced at room temperature for $24 \mathrm{~h}$, heated from $30^{\circ} \mathrm{C}$ to $95^{\circ} \mathrm{C}$ at a heating rate of $10{ }^{\circ} \mathrm{C} / \mathrm{min}$ and determined for transition temperatures (To, Tp, and $\mathrm{Tc}$ ) in a differential scanning calorimeter (TA Instruments, New Castle, USA). The gelatinization enthalpy $(\Delta \mathrm{H})$ was calculated by the software of the instrument.

As described by Hanashiro et al. (1996) [26], the starch samples were dissolved in 50 $\mathrm{mM}$ sodium acetate and debranched with isoamylase (Sigma, Darmstadt, Germany). The degree of polymerization (chain length distribution) was determined and analyzed by HPAEC (Thermo Fisher Scientific, Waltham, USA) via pulsed amperometric detection.

\subsection{Phenotyping of agronomic traits}


The inbred germplasm line GEMS-0067 and its WT control were planted in field randomized complete block design with three replicates. Twenty-one plants were cultivated in three rows $(230 \mathrm{~cm}$ long and $30 \mathrm{~cm}$ apart) per plot. Growth status and disease resistance were observed during all the growth period. All the plants in the middle row of each plot were investigated for anthesis stage, silking stage, growth period, plant height, and ear height. At mature stage, the ears were harvested from the five middle plants in the middle row of each plot, air dried, photographed and investigated for row number per ear, kernel number per row, 100-kernel weight, and kernel weight per plant. And then, their kernels were cut transversely and longitudinally, stained with $\mathrm{I} 2 / \mathrm{KI}$ solution $\left(5 \%[\mathrm{w} / \mathrm{v}] \mathrm{I}_{2} / 10 \%\right.$ [w/v] KI,) as described by Hunt et al. (2013) [40], and photographed under optical microscope (Mshot, Guangzhou, China) with $2 \times$ magnification.

The data was analyzed by Microsoft Excel 2013 (https://www.microsoft.com/) and Origin 8.0 software (https://www.originlab.com/), and presented as means \pm standard deviations (SD). The significance of the difference between the two lines was tested with the method of paired t-test by using SPSS software 11.2 (https://www.ibm.com/analytics/spssstatistics-software).

Author Contributions: Conceptualization, C.X. and F.F.; methodology, N.H.; software, N.H.; investigation, N.H.; resources, C.X.; data curation, W.L.; writing-original draft preparation, N.H.; writing - review and editing, W.L.; supervision, C.X.; funding acquisition, W.L. All authors have read and agreed to the published version of the manuscript.

Funding: This research was funded by Sichuan Science and Technology Program (2018JY0470, 2020YJ0353) and the National Nature Science Foundation of China (32001552).

Conflicts of Interest: The authors declare no conflict of interest.

\section{References}

1. Andorf, C.; Beavis, W.D.; Hufford, M.; Smith, S.; Suza, W.P.; Wang, K.; Woodhouse, M.; Yu, J.; Lubberstedt, T. Technological advances in maize breeding: past, present and future. Theor. Appl. Genet. 2019, 132, 817-849. doi: 10.1007/s00122-019-03306-3

2. Myers, A.M.; Morell, M.K.; James, M.G.; Ball, S.G. Recent progress toward understanding biosynthesis of the amylopectin crystal. Plant Physiol. 2000, 122, 989-997. doi: 10.1104/pp.122.4.989

3. Glaring, M.A.; Koch, C.B.; Blennow, A. Genotype-specific spatial distribution of starch molecules in the starch granule: a combined CLSM and SEM approach. Biomacromolecules 2006, 7, 2310-2320. doi: 10.1021/bm060216e

4. Goren, A.; Ashlock, D.; Tetlow, I.J. Starch formation inside plastids of higher plants. Protoplasma, 2018, 255, 1855-1876. doi:

$$
\text { 10.1007/s00709-018-1259-4 }
$$

5. He, W.; Liu, X.; Lin, L.; Xu, A.; Hao, D.; Wei, C. The defective effect of starch branching enzyme Ilb from weak to strong induces the formation of biphasic starch granules in amylose-extender maize endosperm. Plant Mol. Biol. 2020, 103, 355-371. doi: 10.1007/s11103-020-00998-w

6. Liu, J.; Rong, T.; Li, W. Mutation loci and intragenic selection marker of the granule-bound starch synthase gene in waxy maize. Mol. Breed. 2007, 20, 93-102. doi: 10.1007/s11032-006-9074-6

7. Cai, C.; Lin, L.; Man, J.; Zhao, L.; Wang, Z.; Wei, C. Different structural properties of high-amylose maize starch fractions varying in granule size. J. Agri. Food Chem. 2014, 62, 11711-11721. doi: 10.1021/j5503865e

8. Lin, L.; Guo, D.; Huang, J.; Zhang, X.; Zhang, L.; Wei, C. Molecular structure and enzymatic hydrolysis properties of starches from high-amylose maize inbred lines and their hybrids. Food Hydrocolloids 2016, 58, 246-254. doi: 10.1016/j.foodhyd.2016.03.001

9. Wang, J.; Hu, P.; Chen, Z.; Liu, Q.; Wei, C. Progress in high-amylose cereal crops through inactivation of starch branching enzymes. Front. Plant Sci. 2017, 8, 469. doi: 10.3389/fpls.2017.00469 
10. Raigond, P.; Ezekiel, R.; Raigond, B. Resistant starch in food: a review. J. Sci. Food. Agric. 2015, 95, 1968-1978. doi: 10.1002/jsfa.6966

11. Zhang, X.; Chen, Y.; Zhang, R.; Zhong, Y.; Luo, Y.; Xu, S.; Liu, J.; Xue, J.; Guo, D. Effects of extrusion treatment on physicochemical properties and in vitro digestion of pregelatinized high amylose maize flour. J. Cereal Sci. 2016, 68, 108-115. doi: 10.1016/j.jcs.2016.01.005

12. Song, Y.; Li, X.; Zhong, Y. Optimization of butter, xylitol, and high-amylose maize flour on developing a low-sugar cookie. Food Sci. Nutr. 2019, 7, 3414-3424. doi: 10.1002/fsn3.1160

13. Koch, K.; Gillgren, T.; Stading, M.; Andersson, R. Mechanical and structure properties of solution-cast high-amylose maize starch films. Int. J. Biol. Macromol. 2010, 46, 13-19

14. Jeon, J.S.; Ryoo, N.; Hahn, T.R.; Walia, H.; Nakamura, Y. Starch biosynthesis in cereal endosperm. Plant Physiol. Biochem. 2010, 48, 383-392. doi: 10.1016/j.plaphy.2010.03.006

15. Jiang, L.; Yu, X.; Qi, X.; Yu, Q.; Deng, S.; Bai, B.; Li, N.; Zhang, A.; Zhu, C.; Liu, B.; Pang, J. Multigene engineering of starch biosynthesis in maize endosperm increases the total starch content and the proportion of amylose. Transgenic Res. 2013, 22, 1133e1142. doi: 10.1007/s11248-013-9717-4

16. Zhao, Y.; Li, N.; Li, B.; Li, Z.; Xie, G.; Zhang, J. Reduced expression of starch branching enzyme IIa and Ilb in maize endosperm by RNAi constructs greatly increases the amylose content in kernel with nearly normal morphology. Planta, 2015, 241, 449-461. doi: 10.1007/s00425-014-2192-1

17. Li. C.; Huang, Y.; Huang, R.; Wu, Y.; Wang, W. The gentic architecture of amylose biosyntheses in maize kernel. Plant Biotechnol. J. 2018, 16, 688-695. doi: 10.1111/pbi.12821

18. Guan, H.P.; Preiss, J. Differentiation of the properties of the branching isozymes from maize (Zea mays). Plant Physiol. 1993, 102, 1269-1273. doi: 10.1104/pp.102.4.1269

19. Zhong, Y.; Liu, L.; Qu, J.; Li, S.; Blennow, A.; Seytahmetovna, S.A.; Liu, X.; Guo, D. The relationship between the expression pattern of starch biosynthesis enzymes and molecular structure of high amylose maize starch. Carbohydr. Polym. 2020, 247, 116681. doi: 10.1016/j.carbpol.2020.116681

20. Kim, K.N.; Fisher, D.; Gao, M.; Guiltinan, M. Molecular cloning and characterization of the amylose-extender gene encoding starch branching enzyme IIB in maize. Plant Mol. Biol. 1998, 38, 945-956. doi: 10.1023/a:1006057609995

21. Fuwa, K.; Glover, D.V.; Fujita, A.; Sugimoto, Y.; Inouchi, N. Sturctural and physicochemical properties of endsperm starches possessing diferent alleles at the amyluse-extender and waxy locus in maize (Zea mays L.). Starch, 1999, 51, 147-151. doi: 10.1002/(SICI)1521-379X(199905)51:53.0.CO;2-7

22. Li, L.; Jiang, H.; Campbell, M.; Blanco, M.; Jane, J. Characterization of maize amylose-extender (ae) mutant starches. Part I: Relationship between resistant starch contents and molecular structures. Carbohydr. Polym. 2008, 74, 396-404. doi: 10.1016/j.carbpol.2008.03.012

23. Jiang, H.; Srichuwonga, S.; Campbell, M.; Jane, J.L. Characterization of maize amylose-extender (ae) mutant starches. Part III: Structures and properties of the Naegeli dextrins. Carbohydr. Polym. 2010, 81, 885-891. doi:10.1016/j.carbpol.20

24. Liu, D.; Wellner, N.; Parker, M.L.; Morris, V.J.; Cheng, F. In situ mapping of the effect of additional mutations on starch granule structure in amylose-extender (ae) maize kernels. Carbohydr. Polym. 2015, 118, 199-208. doi: 10.1016/j.carbpol.2014.11.006

25. Campbell, M.R.; Jane, J.L.; Pollak, L.; Blanco, M.; O'Brien, A. Registration of maize germplasm line GEMS-0067. J. Plant Regist. 2007, 1, 60-61. doi: 10.3198/jpr2006.10.0640crg 
26. Hanashiro, I.; Abe, J.; Hizukuri, S. A periodic distribution of the chain length of amylopectin as revealed by high-performance anion-exchange chromatography. Carbohydr. Res. 1996, 283, 151-159. doi: 10.1016/0008-6215(95)00408-4

27. Wang, W.; Guan, L.; Seib, P.A.; Shi, Y.C. Settling volume and morphology changes in cross-linked and unmodified starches from wheat, waxy wheat, and waxy maize in relation to their pasting properties. Carbohydr. Polym. 2018, 196, 18-26. doi.org/10.1016/j.carbpol.2018.05.009

28. Wellner, N.; Georget, D.M.R.; Parker, M.L.; Morris, V.J. In situ Raman microscopy of starch granule structures in wild type and ae mutant maize kernels. Starch 2011, 63, 128-138. doi: 10.1002/star.201000107

29. Liu, D.; Parker, M.L.; Wellner, N.; Kirby, A.R.; Cross, K.; Morris, V.J.; Cheng, F. Structural variability between starch granules in wild type and in ae high-amylose mutant maize kernels. Carbohydr. Polym. 2013, 97, 458-468. doi: 10.1016/j.carbpol.2013.05.013

30. Chen, X.; Du, X.; Chen, P.; Guo, L.; Xu, Y.; Zhou, X. Morphologies and gelatinization behaviours of high-amylose maize starches during heat treatment. Carbohydr. Polym. 2017, 157, 637-642. doi: 10.1016/j.carbpol.2016.10.024

31. Ji, Y.; Pollak, L.M.; Duvick, S.; Seetharaman, K.; Dixon, P.M.; White, P.J. Gelatinization properties of starches from three successive generations of six exotic corn lines grown in two locations. Cereal Chem. 2007, 81, 59-64. doi: 10.1094/CCHEM.2004.81.1.59

32. Singh, S.; Singh, N.; Isono, N.; Noda, T. Relationship of granule size distribution and amylopectin structure with pasting, thermal, and retrogradation properties in wheat starch. J. Agri. Food Chem. 2010, 58, 1180-1188. doi: 10.1021/jf902753f

33. Li, G.; Zhu, F. Amylopectin molecular structure in relation to physicochemical properties of quinoa starch. Carbohydr. Polym. 2017, 164, 396-402. doi: 10.1016/j.carbpol.2017.02.014

34. Zhao, Y.; Li, N.; Li, B.; Li, Z.; Xie, G.; Zhang, J. Reduced expression of starch branching enzyme IIa and IIb in maize endosperm by RNAi constructs greatly increases the amylose content in kernel with nearly normal morphology. Planta, 2015, 241, 449-461. doi: 10.1007/s00425-014-2192-1

35. Zhang, X.D.; Gao, X.C.; Li, Z.W.; Xu, L.C.; Li, Y.B.; Zhang, R.H.; Xu, J.Q.; Guo, D.W. The effect of amylose on kernel phenotypic characteristics, starch-related gene expression and amylose inheritance in naturally mutated high-amylose maize. J. Integrat. Agri. 2020, 19, 1554-1564. doi: 10.1016/S2095-3119(19)62779-6

36. Saghai-Maroof, M.A.; Soliman, K.M.; Jorgensen, R.A.; Allard, R.W. Ribosomal DNA spacer-length polymorphisms in barley: Mendelian inheritance, chromosomal location, and population dynamics. Proc. Natl. Acad. Sci. USA. 1985, 81, 8014-8018. doi: 10.1073/pnas.81.24.8014

37. National Bureau of Standards of the People's Republic of China. Determination of crude starch in cereals seeds. National standards of the people's Republic of China, 1985, GB 5006-85, 1-5.

38. Gibson, T.S.; Solah, V.A.; McCleary, B.V. A procedure to measure amylose in cereal starches and flours with concanavalin A. J. Cereal Sci. 1997, 25, 111-119. doi.org/10.1006/jcrs.1996.0086

39. Wang, J.; Hu, P.; Lin, L.; Chen, Z.; Liu, Q.; Wei, C. Gradually decreasing starch branching enzyme expression is responsible for the formation of heterogeneous starch granules. Plant Physiol. 2018, 176, 582-595. doi: 10.1104/pp.17.01013

40. Hunt, H.V.; Moots, H.M.; Graybosch, R.A.; Jones, H.; Parker, M.; Romanova, O.; Jones, M.K.; Howe, C.J.; Trafford; K. Waxy phenotype evolution in the allotetraploid cereal broomcorn millet: mutations at the GBSSI locus in their functional and phylogenetic context. Mol. Biol. Evol. 2013, 30, 109-122. doi.org/10.1093/molbev/mss209 